10,18

\title{
Пьезоэлектрические свойства 2D наноаллотропов нитрида бора
}

\author{
(С Р.А. Браже, Д.А. Долгов \\ Ульяновский государственный технический университет, \\ Ульяновск, Россия \\ E-mail: brazhe@ulstu.ru \\ Поступила в Редакцию 10 июня 2019 г. \\ В окончательной редакции 10 июня 2019 г. \\ Принята к публикации 8 июля 2019 г.
}

Вычислены независимые компоненты тензора пьезомодулей для различных 2D наноаллотропов нитрида бора. Суть предложенного приближенного метода расчета состояла в том, что эффективный дипольный момент элементарной ячейки 2D структуры, приведенный к единице площади, выражался через тензор упругих жесткостей и относительные деформации ячейки. Показано, что, помимо хорошо известного графеноподобного нитрида бора h-BN, практический интерес могут представлять и другие его гексагональные и тетрагональные наноаллотропы, обладающие более высокими, по сравнению c h-BN, пьезоэлектрическими свойствами.

Ключевые слова: нитрид бора, 2D наноаллотропы, тензор пьезомодулей, независимые компоненты.

DOI: 10.21883/FTT.2019.11.48427.512

\section{1. Введение}

Несмотря на неугасающий интерес к исследованию уникальных упругих свойств графена [1-8] в последние несколько лет взоры многих исследователей обратились к не менее интересному 2D материалу - монослойному нитриду бора в его гексагональной модификации (h-BN) [9-14]. Имея такую же, что и графен (Gr), кристаллическую структуру с почти таким же расстоянием между атомами (1.42 $\AA$ у Gr и $1.45 \AA$ у h-BN), последний, тем не менее, резко отличается от него по своим физическим свойствам.

Это различие связано с тем обстоятельством, что в структуре h-BN атомы трехвалентного бора, чередуясь с атомами пятивалентного азота, образуют не чисто ковалентные $\sigma$-связи, а частично ионные связи из-за смещения электронной плотности к более электроотрицательным атомам азота. Это приводит к разделению свободных и занятых энергетических состояний электронов, в результате чего возникает запрещенная зона шириной $\sim 6 \mathrm{eV}[14]$, и монослойный h-BN, в отличие от $\mathrm{Gr}$, является не полуметаллом, а диэлектриком. Кроме того, при каждом атоме возникают три расположенных под углом $120^{\circ}$ дипольных момента. При этом результирующая поляризация 2D структуры равна нулю, но при ее механических деформациях равновесие нарушается и возникает отличная от нуля поляризация. Таких образом, монослойный h-BN, как и другие 2D соединения типа $\mathrm{A}^{\mathrm{III}} \mathrm{B}^{\mathrm{V}}$ (BP, AlN, AlP, AlAs, $\mathrm{GaN}, \mathrm{GaP}, \mathrm{GaAs}$ и др.) должны быть пьезоэлектриками, что открывает широкие возможности их применения в наноэлектронике и наноэлектромеханических системах управления информацией. Использование естественных 2D пьезоэлектриков типа h-BN на практике значительно проще, чем искусственное придание пьезоэлектрических свойств графену путем нарушения центросимметричности его структуры перфорированием или допированием [15-18].

Целью настоящей работы является оценка численных значений независимых компонент тензора пьезомодулей для 2D наноаллотропов BN. K ним относится не только структурный аналог классического h-BN, но и аналоги теоретически предсказанных [19-21] додекатриграфена $(\mathrm{C})_{63(12)}$, додекагексатетраграфена $(\mathrm{C})_{664}$ и октатетраграфена (октаграфена) (C) 44 .

\section{2. Исследуемые структуры и методы расчета}

Все исследуемые структуры представлены на рисунке. В частности, для структуры $(\mathrm{BN})_{6}$ показано, что приложение внешнего электрического поля напряженности $\mathbf{E}$ в направлении оси $x_{2}$ приводит к деформации растяжения ячеек за счет смещения подрешеток бора и азота в противоположные стороны вдоль той же оси (прямой пьезоэффект). Наоборот, такая деформация приводит к отличному от нуля суммарному дипольному моменту в этом же направлении (обратный пьезоэффект). Аналогично, в случае структуры $(\mathrm{BN})_{44}$ внешнее электрическое поле, приложенное в направлении оси $x_{1}$, вызывает такой же эффект, а поле, приложенное вдоль оси $x_{2}$ приводит к деформации сдвига в плоскости $\left(x_{1}, x_{2}\right)$.

Величина соответствующего пьезомодуля $d_{22}$, описывающего рассмотренный эффект, была оценена в работе [14] путем DFT-вычислений и дала результат $d_{22}=0.55 \cdot 10^{-12} \mathrm{C} / \mathrm{N}$. Следует отметить, что недостатком метода DFT при его применении к новым структурам является невозможность адекватного зада- 

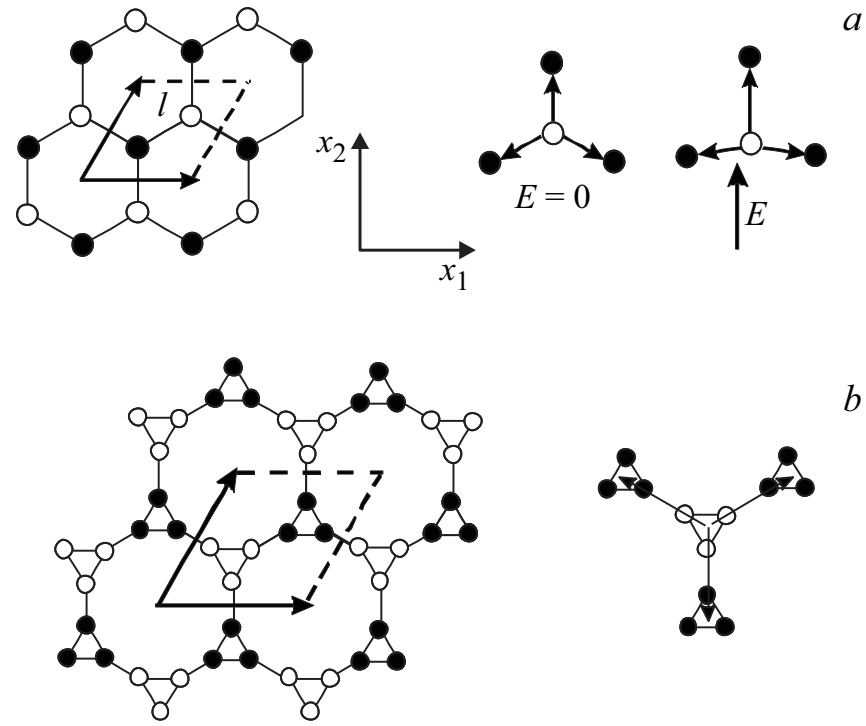

$b$

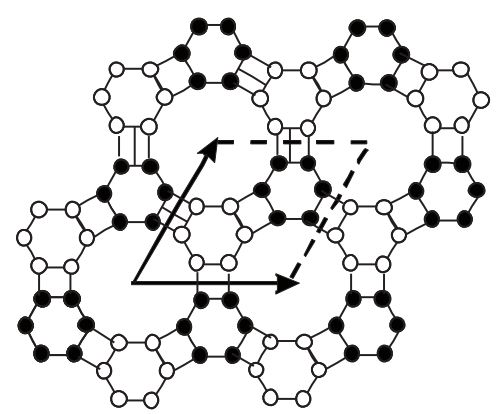

$c$
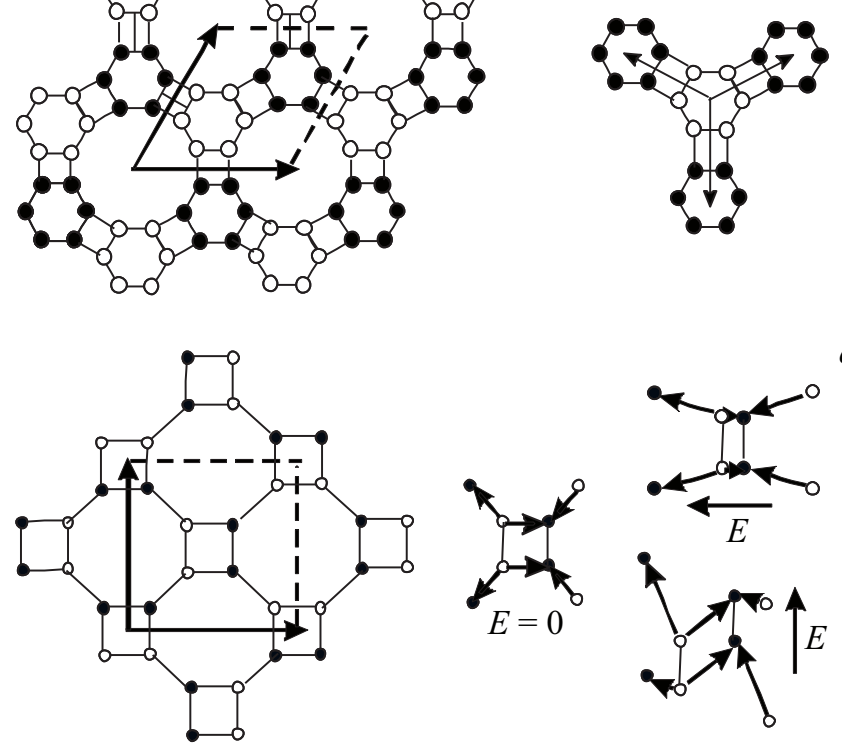

Атомная структура, элементарная ячейка и схема дипольных моментов 2D наноаллотропов нитрида бора: классический гексагональный нитрид бора $(\mathrm{BN})_{6}$ или h-BN $(a)$, додекатринитрид бора $(\mathrm{BN})_{63(12)}(b)$, додекагексатетранитрид бора $\mathrm{BN}_{664}(c)$, и октатетранитрид бора $\mathrm{BN}_{44}(d)$. Атомы бора и азота показаны темными и светлыми точками соответственно. Оси $x_{1}, x_{2}$, кристаллофизической системы координат соответствуют выбранной ориентации структуры.

ния эффективного потенциала, в котором двигаются взаимодействующие валентные электроны, рассматриваемые как свободные (формализм Кона-Шэма). Поэтому невозможно оценить погрешность расчета без сравнения его с результатами, полученными на основе других методов или экспериментальным путем. Здесь мы предлагаем аналитический метод нахождения компонент тензора пьезомодулей $d_{i j k}$, основанный на выражении вектора поляризации $P_{i}$ через тензор механических напряжений $t_{j k}$

$$
P_{i}=d_{i j k} t_{j k},
$$

который, в свою очередь, в пределах закона Гука пропорционален относительной деформации

$$
t_{j k}=\frac{1}{2} c_{j k l m}\left(\frac{\partial u_{l}}{\partial x_{m}}+\frac{\partial u_{m}}{\partial x_{l}}\right),
$$

где $u_{l}, u_{m}-$ смещения по координатам $x_{m}, x_{l}$ соответственно $(j, k, l, m=1,2)$.

Закон Гука допускает возможность, лишь малых статических, либо гармонических деформаций элементарной ячейки в кристаллической структуре, когда $c_{j k l m}$ являются упругими жесткостями второго порядка (в матричном представлении). В случае статических деформаций мы имеем изотермические значения $c_{j k l m}$, а в случае гармонических деформаций - адиабатические значения $c_{j k l m}$, которые обычно превышают изотермические значения. Превышение будет тем более заметным, если колебания становятся ангармоническими, когда следует учитывать упругие жесткости третьего порядка и уравнение (2) переходит в нелинейное. Для многих практических приложений пьезоэффекта вполне достаточно знания упругих и пьезоэлектрических констант, измеренных в статическом режиме.

Наноаллотропы $(\mathrm{BN})_{6},(\mathrm{BN})_{63(12)},(\mathrm{BN})_{664}$ принадлежат к классу симметрии $3 m$, а $(\mathrm{BN})_{44}$ - к классу $m$. Матрицы упругих жесткостей $\left(c_{i j}\right)$ и пьезомодулей $\left(d_{i j}\right)$ для них имеет вид [17]:

класс $3 m$

класс $m$

$$
\begin{aligned}
& \left(\begin{array}{ccc}
c_{11} & c_{12} & 0 \\
c_{12} & c_{11} & 0 \\
0 & 0 & \frac{1}{2}\left(c_{11}-c_{12}\right)
\end{array}\right)_{(2)}, \quad\left(\begin{array}{ccc}
c_{11} & c_{12} & 0 \\
c_{12} & c_{11} & 0 \\
0 & 0 & c_{33}
\end{array}\right)_{(3)} \\
& \left(\begin{array}{ccc}
0 & 0 & -2 d_{22} \\
-2 d_{22} & d_{22} & 0
\end{array}\right)_{(1)}, \quad\left(\begin{array}{ccc}
d_{11} & -d_{11} & 0 \\
0 & 0 & d_{23}
\end{array}\right)_{(2)} .
\end{aligned}
$$

Матричные обозначения тензоров $c_{j k l m}$ и $d_{i j k}$ получены путем свертки по парам симметричных индексов в следующем виде: $11 \rightarrow 1 ; 22 \rightarrow 2 ; 12,21 \rightarrow 3$. В круглых скобках справа от матриц указано число независимых компонент соответствующего тензора.

Производя в (1), (2) суммирование по повторяющимся индексам с учетом вида матриц $c_{i j}$ и $d_{i j}$, получаем для класса $3 m$

$$
P_{2}=2 d_{22}\left(c_{11}-c_{12}\right) \frac{\partial u_{2}}{\partial x_{2}}
$$

для класса $m$

$$
\begin{gathered}
P_{1}=2 d_{11}\left(c_{11}-c_{12}\right) \frac{\partial u_{1}}{\partial x_{1}} \\
P_{2}=2 d_{23} c_{33} \frac{\partial u_{1}}{\partial x_{2}}
\end{gathered}
$$


Таблица 1. Геометрические параметры, определяющие значения пьезомодулей 2D наноаллотропов нитрида бора

\begin{tabular}{l|c|c|c}
\hline Параметр & $Z$ & $l$ & $S$ \\
\hline$(\mathrm{BN})_{6}$ & $1 / 3$ & $a_{\mathrm{BN}}$ & $\frac{3 \sqrt{3}}{2} a_{\mathrm{BN}}^{2}$ \\
\hline$(\mathrm{BN})_{63(12)}$ & 1 & $\left(1+\frac{2 \sqrt{3}}{3}\right) a_{\mathrm{BN}}$ & $\frac{3 \sqrt{3}}{2}\left(1+\frac{2 \sqrt{3}}{2}\right) a_{\mathrm{BN}}^{2}$ \\
\hline$(\mathrm{BN})_{664}$ & 2 & $(1+\sqrt{3}) a_{\mathrm{BN}}$ & $\frac{3 \sqrt{3}}{2}(1+\sqrt{3}) a_{\mathrm{BN}}^{2}$ \\
\hline$(\mathrm{BN})_{44}$ & 2 & $a_{\mathrm{BN}}$ & $(2+\sqrt{2})^{2} a_{\mathrm{BN}}^{2}$
\end{tabular}

С другой стороны, полный дипольный момент, связанный с деформацией элементарной ячейки 2D кристаллической структуры, приходящийся на единицу площади, в случае наноаллотропов класса $3 m$,

$$
P_{2}=\frac{Z e l}{S} \frac{\partial u_{2}}{\partial x_{2}}
$$

где $Z$ - доля заряда одного знака, приходящегося на элементарную ячейку и участвующего в создании одного дипольного момента, $e$ - элементарный заряд, $l$ - расстояние между центрами положительного и отрицательного зарядов (длина диполя), $S$ - площадь элементарной ячейки. Для наноаллотропов класса $m$ имеем

$$
\begin{aligned}
& P_{1}=\frac{Z e l}{S} \frac{\partial u_{1}}{\partial x_{1}}, \\
& P_{2}=\frac{Z e l}{S} \frac{\partial u_{1}}{\partial x_{2}} .
\end{aligned}
$$

Приравнивая правые части выражений (3)-(5) и $(6)-(8)$ для соответствующих классов симметрии, получаем

для класса $3 m$

$$
d_{22}=\frac{1}{2} \frac{Z e l}{S\left(c_{11}-c_{12}\right)}
$$

а для класса $m$

$$
\begin{gathered}
d_{11}=\frac{1}{2} \frac{Z e l}{S\left(c_{11}-c_{12}\right)}, \\
d_{23}=\frac{1}{2} \frac{Z e l}{S c_{33}} .
\end{gathered}
$$

Выражения для параметров $Z, l, S$ исследуемых наноаллотропов нитрида бора представлены в табл. 1. Здесь $a_{\mathrm{BN}}-$ расстояние между атомами бора и азота.

Наиболее сложным моментом в расчете пьезомодулей исследуемых структур, в особенности, если они еще не получены экспериментально, является правильный выбор метода отыскания значений соответствующих компонент тензоров упругих жесткостей $c_{i j}$ для их подстановки в формулы (9)-(11). Выше мы уже отмечали, что здесь нас, в первую очередь, интересуют статиче- ские значения упругих констант, поскольку расчет их динамических значений требует учета многочисленных поправок, выбор которых довольно субъективен.

В связи с этим в данной работе мы ограничиваемся методом связывающих орбиталей Харрисона, модифицированным Давыдовым [22-24] для 2D структур, в том числе двухатомных с ковалентно-полярной связью. Этим методом мы находим константы центрального и нецентрального взаимодействия, которые затем используем в модели Китинга [25] по схеме, приложенной в [2], для нахождения упругих жесткостей второго порядка по формулам [2,26]

$$
\begin{aligned}
& c_{11}=\frac{1}{\sqrt{3}}\left(4 \alpha+\beta+18 \frac{\alpha \beta}{4 \alpha+\beta}\right), \\
& c_{12}=\frac{1}{\sqrt{3}}\left(4 \alpha+\beta-18 \frac{\alpha \beta}{4 \alpha+\beta}\right)
\end{aligned}
$$

для класса симметрии $3 m$ и

$$
\begin{gathered}
c_{11}=\frac{4(2 \alpha+3 \beta)}{(1+\sqrt{2})^{2}}, \quad c_{12}=\frac{4(2 \alpha-\beta)}{(1+\sqrt{2})^{2}}, \\
c_{11}=\frac{2 \alpha+\beta}{(1+\sqrt{2})^{2}}
\end{gathered}
$$

для класса симметрии $m$. Здесь $\alpha$ и $\beta-$ константы центрального и нецентрального взаимодействия, которые мы приравняли соответственно $k_{0}$ и $k_{1}$ из работ Давыдова [27,28]. В работе [28] также введены относительные (по отношению к графену) константы соответствующих взаимодействий $K_{0}=k_{0}(\mathrm{AB}) / k_{0}(\mathrm{Gr})$ и $K_{1}=k_{1}(\mathrm{AB}) / k_{3}(\mathrm{Gr})$ для двухатомных $2 \mathrm{D}$ структур типа $\mathrm{A}^{Ш} \mathrm{~B}^{\mathrm{V}}$. Для $\mathrm{BN} k_{0}=0.80, k_{1}=0.65$. Используя эти значения для графенов $(\mathrm{C})_{63(12)},(\mathrm{C})_{664}$ и $(\mathrm{C})_{44}$, для которых нами ранее были вычислены $\alpha$ и $\beta$ [29], мы можем найти силовые константы также для 2D наноаллотропов нитрида бора $(\mathrm{BN})_{63(12)},(\mathrm{BN})_{664},(\mathrm{BN})_{44}$ и завершить задачу.

В табл. 2 представлены результаты оценочных расчетов силовых констант, независимых компонент матриц упругих жесткостей и пьезомодулей исследуемых 2D наноаллотропов нитрида бора.

\section{3. Полученные результаты и их обсуждение}

Анализируя полученные результаты, обратим внимание на то, что вычисленное нами из простых физических соображений значение пьезомодуля $d_{22}$ для h-BN, или $(\mathrm{BN})_{6}$ в наших обозначениях, практически совпадает с результатом, полученным в работе [14]. Это говорит, во-первых, о достоверности результатов и, во-вторых, о пригодности предложенного метода для оценки пьезоэлектрических свойств других, в том числе еще не синтезированных, а „нарисованных“ $2 \mathrm{D}$ наноаллотропов других $\mathrm{A}^{\mathrm{III}} \mathrm{B}^{\mathrm{V}}$ соединений. 
Таблица 2. Оценочные значения упругих и пьезоэлектрических характеристик 2D наноаллотропов нитрида бора

\begin{tabular}{c|c|c|c|c}
\hline Параметр & $(\mathrm{BN})_{6}$ & $(\mathrm{BN})_{63(12)}$ & $(\mathrm{BN})_{664}$ & $(\mathrm{BN})_{44}$ \\
\hline$a_{\mathrm{BN}}$ & 1.45 & 1.6 & 1.44 & 1.38 \\
\hline$\alpha, \mathrm{N} / \mathrm{m}$ & 141 & 20.1 & 96.0 & 142 \\
\hline$\beta, \mathrm{N} / \mathrm{m}$ & 25.3 & 3.77 & 17.9 & 26.5 \\
\hline$c_{11}, \mathrm{~N} / \mathrm{m}$ & 405 & 100 & 277 & 250 \\
\hline$c_{12}, \mathrm{~N} / \mathrm{m}$ & 279 & 39.3 & 188 & 141 \\
\hline$c_{33}, \mathrm{~N} / \mathrm{m}$ & - & - & - & 62.6 \\
\hline$d_{22}, 10^{-12} \mathrm{C} / \mathrm{N}$ & 0.56 & 0.68 & 0.321 & - \\
\hline$d_{11}, 10^{-12} \mathrm{C} / \mathrm{N}$ & - & - & - & 0.92 \\
\hline$d_{23}, 10^{-12} \mathrm{C} / \mathrm{N}$ & - & - & - & 1.60
\end{tabular}

Примеч ание. Значения длины межатомной связи $a_{\mathrm{NB}}$ для $(\mathrm{BN})_{664}$ и $(\mathrm{BN})_{44}$ взяты из работы [30], а для $(\mathrm{BN})_{63(12)}$ принято ориентировочные значения $1.6 \AA$, исходя из факта, что для $2 \mathrm{D}$ наноаллотропов углерода и нитрида бора $a_{\mathrm{CC}} \approx a_{\mathrm{BN}}$ с точностью до второй значащей цифры.

Для оценки статических, а так же квазигармонических значений пьезоэлектрических коэффициентов 2D наноаллотропов целесообразно использовать значения упругих жесткостей, вычисленные без вызывающих дополнительную жесткость поправок на энергию отталкивания, приходящуюся на одну связь, образованную орбиталями соседних атомов [24], при расчете силовых констант ковалентных связей и ангармонические эффекты.

Как видно из табл. 2 , из рассмотренных $2 \mathrm{D}$ наноаллотропов нитрида бора несколько большими значениями пьезомодулей по сравнению с классическим монослойным h-BN должны обладать бор-азотные аналоги додекатриграфена $(\mathrm{BN})_{63(12)}$ и октаграфена $(\mathrm{BN})_{44}$. В частности, у последнего материала пьезомодуль $d_{22}$ превышает пьезомодуль $d_{22}$ h-BN в 1.6 раза, а пьезомодуль $d_{23}-$ в 2.9 раза. Кроме того, пьезомодуль $d_{23}$ соответствует сдвиговым деформациям в плоскости $\left(x_{1}, x_{2}\right)$ при приложении электрического поля вдоль оси $x_{2}$. Это обстоятельство позволяет использовать монослойный $(\mathrm{BN})_{44}$ для изготовления пьезоэлектрических преобразователей в наноакустоэлектронных линиях задержки на сдвиговых упругих волнах. В связи с изложенным, целесообразно активизировать усилия по синтезу данного материала и его практическому использованию в наноэлектронике, пьезоэлектрических датчиках и наноэлектромеханических системах.

\section{4. Заключение}

Основным результатом работы является предложенный авторами аналитический подход к оценочным расчетам независимых компонент тензора пьезомодулей $2 \mathrm{D}$ наноаллотропов бинарных соединений типа $\mathrm{A}^{\mathrm{III}} \mathrm{B}^{\mathrm{V}}$ различной симметрии, в частности, нитрида бора. Данная методика позволяет без больших затрат машинного времени на ab initio расчеты получить адекватные значения пьезоэлектрических констант для двумерных кристаллических решеток, образованных химическими соединениями указанного типа, даже если они еще не получены экспериментально, а сконструированы пока лишь теоретически. Это дает возможность осуществлять целенаправленный синтез наиболее перспективных с точки зрения практических приложений $2 \mathrm{D}$ наноаллотропов определенной симметрии. Из монослойных наноаллотропов нитрида бора таковым в ближайшем будущем может стать бор-азотный аналог октаграфена $(\mathrm{BN})_{44}$, обладающий по нашим оценкам более сильным пьезоэффектом, чем классический монослойный h-BN. Более того, в нем возможны как продольный, так и поперечный пьезоэффекты.

\section{Конфликт интересов}

Авторы заявляют, что у них нет конфликта интересов.

\section{Список литературы}

[1] A.H. Castro Neto, E. Guinea, N.M.R. Peres, K.S. Novoselov, A.K. Geim. Rev. Mod. Phys. 81, 109 (2009).

[2] С.Ю. Давыдов. ФТТ 52, 756 (2010).

[3] V.M. Pereira, R.M. Ribeiro, N.M.R. Peres, A.H. Castro Neto. Europhys. Lett. 92, 67001 (2011).

[4] J.U. Lee, D. Yoon, H. Kim, S.W. Lee, H. Cheong. Phys. Rev. B 83, 081419 (2011).

[5] Р.А. Браже, В.С. Нефедов. ФТТ 56, 602 (2014).

[6] Р.А. Браже, Д.Е. Дулов. ФТТ 56, 2490 (2014).

[7] Р.А. Браже, М.В. Литвиненко. ЖТФ 85, 118 (2015).

[8] Р.А. Браже, Р.М. Мефтахутдинов. ЖТФ 86, 112 (2016).

[9] Q. Peng, J. Wei, S. De. Comput. Mater. Sci. 56, 11 (2012).

[10] X. Zhao, L. Li, M. Zhao. J. Phys.: Condens. Matter. 26, 095002 (2014).

[11] T. Han, Y. Luo, C. Wang. J. Phys.D. 47, 025303 (2014).

[12] X. Li, J. Zhou, W. Gao. Nanotechnology 25, 105701 (2014).

[13] M.J.S. Matos, M.S.C Mazzoni, H. Chacham. Nanotechnology 25, 165705 (2014).

[14] M. Droth, G. Burkard, V.M. Pereira. Phys. Rev. B 94, 075404 (2016).

[15] S. Chandratre, P. Sharma. Appl. Phys. Lett. 100, 023114 (2012).

[16] M.T. Ong, E.J. Reed. FCS Nano 6, 1387 (2012).

[17] Р.А. Браже, А.И. Кочаев, А.А. Советкин. ФТТ 55, 1809 (2013).

[18] Р.А. Браже, А.И. Кочаев, А.А. Советкин. ФТТ 55, 1979 (2013).

[19] A.N. Enyashin, A.L. Ivanovskii. Phys. Status Solidi B 8, 1879 (2011).

[20] Е.А. Беленков, В.А. Грешняков. ФТТ 55, 8, 1640 (2013).

[21] А.И. Подливаев, Л.А. Опенов, ФТТ 55, 2464 (2013).

[22] С.Ю. Давыдов, А.А. Лебедев, Н.Ю. Смирнова. ФТТ 51, 452 (2009). 
[23] С.Ю. Давыдов. ФТТ 51, 2041 (2009).

[24] С.Ю. Давыдов, О.В. Посредник. ФТТ 57, (2015).

[25] P.N. Keating. Phys. Rev. 145, 637 (1966).

[26] Р.А. Браже, А.И. Кочаев, Р.М. Мефтахутдинов. ФТТ 53, 1614 (2011).

[27] С.Ю. Давыдов. ФТТ 52, 172 (2010).

[28] С.Ю. Давыдов. ФТТ 58, 392 (2016).

[29] Р.А. Браже, А.А. Коренин, А.И. Кочаев, Р.М. Мефтахутдинов. ФТТ 53, 1406 (2011).

[30] Р.А. Браже, А.А. Каренин. Изв. вузов. Поволжский регион. Физ.-мат. науки 18, 105 (2011).

Редактор К.В. Емиев 